\title{
Avaliação do conhecimento sobre HIV/AIDS e uso de preservativo em um grupo de idosos da Cidade de São Paulo
}

\author{
HIV/AIDS knowledge evaluation and condom use in an elderly group \\ in the City of São Paulo
}

\begin{abstract}
Bruna Martins Liberali ${ }^{1}$, Stella Carvalho Moreno das Neves ${ }^{1}$, Lais dos Santos de Oliveira ${ }^{1}$, Bruno Dias Batista ${ }^{1}$, Daniela Cristina Feliciano Ferreira Nacaratto ${ }^{1}$, Cinthya Luzia Cavazzana ${ }^{2}$
\end{abstract}

Liberali BM, Neves SCM, Oliveira LS, Batista BD, Nacaratto DCFF, Cavazzana CL. Avaliação do conhecimento sobre HIV/AIDS e uso de preservativo em um grupo de idosos da Cidade de São Paulo / HIV/AIDS knowledge evaluation and condom use in an elderly group in the City of São Paulo. Rev Med (São Paulo). 2020 mar.-abr.;99(2):104-8.

RESUMO: Introdução: O processo de transição demográfica no Brasil vem sendo acompanhado de um aumento na incidência de HIV em idosos, fator esse que deve ser investigado a fim de encontrar possíveis condições relacionadas, permitindo a criação de estratégias eficazes para a redução de tal incidência. Objetivos: Avaliar o conhecimento sobre HIV/AIDS e o uso de preservativo em um grupo de idosos da cidade de São Paulo. Métodos: Estudo analítico, transversal e quantitativo realizado a partir da aplicação do "Questionário sobre conhecimento e prevenção pessoal de HIV/AIDS“ em 115 idosos de ambos os sexos com idade igual ou superior a 60 anos, orientados em tempo e espaço e que aceitaram os termos do TCLE. A análise de dados foi feita de forma quantitativa, sob os aspectos: conhecimento sobre HIV/AIDS (conceito, transmissão e prevenção) e prevenção pessoal. Os dados foram analisados por estatística descritiva e inferencial pelo programa R 3.6.0. Resultados: Houve predominância de respondentes do sexo feminino (68,7\%). A maioria dos entrevistados não faz o uso de preservativo (75\%). Apesar de $83 \%$ apresentarem conhecimento suficiente sobre HIV/AIDS, $66 \%$ acreditam que existe cura para a doença e $54 \%$ afirmaram que o vírus pode ser transmitido por picada de mosquito. Conclusões: constatou-se que, apesar do bom nível de conhecimento demonstrado pelos participantes sobre o HIV/AIDS, ainda persistem dúvidas quanto às formas de transmissão e possibilidade de cura da doença. Demonstra-se a necessidade de compreensão do processo de expansão do HIV/ AIDS pelos profissionais de saúde e que a população acima de 60 anos entenda como a doença funciona, seus mecanismos de transmissão e prevenção.

Descritores: HIV; Síndrome de imunodeficiência adquirida; Idoso; Preservativos, Conhecimento; Brasil/etnologia.

\begin{abstract}
Introduction: The process of demographic transition in Brazil has been followed by an increase in the incidence of HIV in the elderly, which should be investigated in order to find out possible related conditions, allowing the creation of effective strategies to reduce such incidence. Objectives: This study has the objective of evaluating the knowledge about HIV/SIDA and condom use in a group of elderly people in the city of São Paulo, to verify if there is a relation between these factors and the increased incidence of HIV/SIDA in this age group. Methods: The analytical, cross-sectional and quantitative study was conducted by the application of the "Questionnaire about knowledge and personal prevention of HIV / AIDS" in 115 elderly men and women aged 60 years and over, oriented in time and space and who accepted the terms of WICF (Written Informed Consent Form). The data analysis was performed quantitatively, under the following aspects: knowledge about HIV/AIDS (concept, transmission and prevention) and personal prevention. The data were analyzed by descriptive and inferential statistics by the R 3.6.0 software. Results: There was a predominance of female respondents $(68,7 \%)$. Most respondents do not use condoms (75\%). Although $83 \%$ have sufficient knowledge of HIV/SIDA, $66 \%$ believe there is a cure for the disease and $54 \%$ said that the virus could be transmitted by mosquito bites. Conclusions: it was found that despite the good level of knowledge shown by participants about HIV/ SIDA, questions still persist about the ways of transmission and the possibility of cure of the disease. It demonstrates the need of understanding about the spread process of HIV/AIDS by health professionals and that the population over 60, understands how the disease works, its mechanisms of transmission and prevention.
\end{abstract}

Keywords: HIV; Acquired immunodeficiency syndrome; Aged; Condoms; Knowledge; Brazil/ethnology.

\footnotetext{
1. Universidade Anhembi Morumbi. ORCID iD: Liberali BM - https://orcid.org/0000-0001-8907-3094; Neves SCM - https://orcid.org/0000-0002-4181548X; Oliveira LS - https://orcid.org/0000-0002-1013-1501. Batista BD - https://orcid.org/0000-0002-8395-1439; Nacaratto DCFF - https://orcid. org/0000-0002-7569-6503. E-mail: brunaliberali@gmail.com, ste_cmn@hotmail.com, laissoliv@gmail.com,brunodiass@terra.com.br, nacaratto@ gmail.com.

2. Secretaria Municipal de Saúde. ORCID iD: https://orcid.org/0000-0002-5088-4831. Email: cicavazzana@yahoo.com.br.

Endereço para correspondência: Bruna Martins Liberali. Rua Ipanema, 67, apto. 133 bloco B. Mooca, São Paulo, SP. CEP: 03164-200. Email: brunaliberali@gmail.com
} 


\section{INTRODUÇÃO}

To Brasil, o acentuado declínio da fecundidade associado à redução da mortalidade resultou em um processo de envelhecimento populacional significativo. Em 2010 os idosos representavam 8,6\% da população nacional, tendo como projeção representarem $20 \%$ da população em 2050. A área da saúde enfrenta mudanças importantes decorrentes dessa transição demográfica, como o aumento da prevalência de doenças crônicas ${ }^{1}$.

Um dos desafios decorrentes da transição demográfica é o aumento da incidência de HIV (Human Immunodeficiency Virus) em idosos ao longo dos anos, uma vez que tem sido verificado o aumento do número de infectados nessa faixa etária, apesar da maioria dos casos de infecção pelo HIV acometer jovens de 25 a 39 anos $^{2}$. Pela análise dos dados epidemiológicos nacionais, no Brasil, até 2007 foram notificados pelo Sinan 168 casos de HIV em indivíduos com 60 anos ou mais, enquanto que, em 2018 houve notificação de 627 novos casos, representando um total de 7.469 casos notificados entre 2007 e $2018^{3}$.

Existem diversos aspectos que relacionam a vulnerabilidade dos idosos ao HIV, tais como, despreparo dos profissionais de saúde quanto à sexualidade do idoso, pragmatismos sociais referentes à atividade sexual na terceira idade, a baixa aderência ao uso de preservativos, e a falta de informação adequada sobre HIV/AIDS (Human Immunodeficiency Virus/Acquired Immunodeficiency Syndrome) e outras ISTs (Infecções Sexualmente Tranmissíveis).

O Ministério da $\mathrm{Saúde}^{4}$, através da Política Nacional do Idoso busca combater o HIV/AIDS por meio de campanhas, distribuição de preservativos e palestras sobre seu uso correto, a fim de educar, esclarecer dúvidas da população idosa e previni-la do HIV. Para Souza et al. ${ }^{5}$, apesar do desenvolvimento de tais campanhas, essas ainda são desconhecidas por muitos, fato que colabora para a deficiência na disseminação do conhecimento das ISTs.

O aumento no número de casos de HIV/AIDS em idosos pode estar associado aos avanços da indústria farmaceutica e da medicina que permitem o prolongamento da vida sexual ativa ${ }^{6}$. Além disso, a negligência quanto ao uso de preservativos nas relações sexuais e a falta de conhecimento sobre a doença, sua transmissão e prevenção também podem estar relacionados.

$\mathrm{O}$ presente estudo tem como objetivo avaliar o conhecimento sobre HIV/AIDS e o uso de preservativos em um grupo de idosos da cidade de São Paulo, para verificar se há relação de tais fatores com o aumento da incidência de HIV/AIDS nessa faixa etária. A finalidade do estudo é reconhecer se há correlação da utilização de preservativo com o nível de conhecimento da doença.

\section{MÉTODOS}

Trata-se de um estudo analítico, transversal e quantitativo realizado a partir da aplicação do questionário "Questionário sobre conhecimento e prevenção pessoal de HIV/AIDS" em 115 idosos de ambos os sexos, no Centro Integrado de Saúde da Universidade Anhembi Morumbi de São Paulo, em maio de 2019. A realização do estudo foi aprovada pelo Comitê de Ética em Pesquisa da Universidade Anhembi Morumbi (CAAE: 05032818.9.0000.5492).

O questionário “Questionário sobre conhecimento e prevenção pessoal de HIV/AIDS" foi criado pelos autores do estudo, sendo uma adaptação do "Questionário de HIV na Terceira Idade" (QHIV3I). Tal adaptação foi realizada pois o QHIV3I avalia além do conhecimento de idosos sobre HIV/AIDS, características específicas dos participantes que não foram levadas em consideração no presente estudo (nível socioeconômico, escolaridade, presença de parceiro fixo e religião). A adaptação consistiu na seleção de 9 das 15 questões do QHIV3I, e na introdução de uma questão sobre prevenção sexual.

$\mathrm{O}$ questionário sobre conhecimento de $\mathrm{HIV} /$ AIDS abrange questões sobre o conhecimento geral da doença (perguntas 1 à 5), transmissão (perguntas 6 à 8) e prevenção (pergunta 9) de HIV/AIDS. As perguntas foram respondidas em "sim", "não", ou "não sei”. Possui ainda uma décima questão sobre prevenção pessoal "você faz uso de camisinha nas relações sexuais?" com duas opções de resposta, "sim" ou "não".

Os critérios de inclusão foram: idosos com idade igual ou superior a 60 anos, de ambos os sexos, orientados em tempo e espaço e que aceitaram os termos do TCLE. Os critérios de exclusão foram: indivíduos não alfabetizados e/ou incapacitados de responderem o questionário.

O questionário foi aplicado através de busca ativa de idosos que preenchessem os critérios de inclusão e que encontravam-se no Centro Integrado de Saúde da Universidade Anhembi Morumbi durante a realização da pesquisa (maio de 2019). Antes de responderem o questionário, os idosos foram abordados e informados da natureza do estudo, e, ao concordarem em participar da pesquisa, assinaram um termo de consentimento livre esclarecido (TCLE).

A análise de dados foi feita de forma quantitativa, sob os aspectos: conhecimento sobre HIV/AIDS, e prevenção pessoal. O conhecimento da doença foi examinado a partir das perguntas 1 à 9 , sobre seu conceito, sua transmissão e sua prevenção. Seis questões corretas ou mais indicam que o entrevistado possui conhecimento sobre a doença, e qualquer resultado diferente deste indica conhecimento insuficiente sobre HIV/AIDS. A resposta "não sei" foi considerada incorreta, pois indica falta de conhecimento sobre o assunto.

E por fim, a pergunta sobre prevenção pessoal indicou se os entrevistados fazem uso de preservativos 
durante as relações sexuais.

Para analisar os dados levantados foi utilizada a estatística descritiva de frequência e percentual de ocorrência de cada classe para as variáveis categóricas. Para verificar a associação entre as variáveis categóricas foi utilizado o teste do qui-quadrado de Pearson com correção de continuidade de Yates. Para as variáveis "média de acerto das nove questões" foi utilizado o teste F para verificar as diferenças entre indivíduos que usam preservativo ou não. Todas as análises estatísticas foram realizadas no software R 3.6.0 ( $\mathrm{R}$ Development Core Team, 2018).

\section{RESULTADOS}

O estudo avaliou 79 mulheres e 36 homens, sendo $68,7 \%$ do sexo feminino e $31,3 \%$ do sexo masculino. A média das idades dos respondentes é igual a $68 \pm 6,3$. Não foi evidenciada associação entre o sexo dos respondentes e o conhecimento sobre a doença $(\mathrm{p}=0,999)$, dado que $84,6 \%$ das mulheres possuem conhecimento suficiente, e $83,3 \%$ dos homens também. Na dimensão sobre conhecimentos gerais do HIV, ambos os sexos obtiveram a mesma taxa de acerto (74\%). Sobre a transmissão, as mulheres apresentaram maior taxa de acerto (86\%) que os homens (80\%), apesar desta diferença não apresentar significância estatística $(\mathrm{p}=0,133)$. Quanto prevenção, a diferença da taxa de acerto foi de apenas $1 \%$, sendo $89 \%$ para as mulheres e $90 \%$ para os homens.

Em uma análise geral, a média de acertos no quesito "conhecimento geral" foi de $74 \%$, enquanto que na transmissão foi de $82 \%$ e na prevenção $90 \%$. A maior defasagem no âmbito sobre conhecimento geral da doença foi sobre a presença dos sintomas nos indivíduos com HIV (taxa de acerto de 44\%), seguida da existência de cura para AIDS (taxa de acerto de 66\%). Já na dimensão sobre transmissão da doença, a maior defasagem foi sobre a possibilidade do vírus HIV ser transmitido por picada de mosquito (taxa de acerto de 54\%) (Tabela 1).

Foi verificado que 96 respondentes possuem conhecimento suficiente sobre HIV/AIDS (83\%), e 19 respondentes possuem conhecimento insuficiente (17\%). A distribuição dos acertos nas subcategorias pode ser observada na Tabela 2.

Tabela 1. Taxa de acerto por pergunta do questionário

\begin{tabular}{l|l|c}
\hline Dimensão avaliada & Pergunta & Resposta correta \\
\hline \multirow{5}{*}{ Conhecimento geral } & 1. O vírus HIV é o causador da AIDS? & $87 \%$ \\
\cline { 2 - 3 } & 2. A pessoa com o vírus da AIDS sempre apresenta os sintomas da doença? & $44 \%$ \\
\cline { 2 - 3 } & e usuários(as) de drogas? & $78 \%$ \\
\cline { 2 - 3 } & $\begin{array}{l}\text { 4. Os indivíduos da terceira idade não devem se preocupar com a AIDS, pois ela } \\
\text { atinge apenas os jovens? }\end{array}$ & $96 \%$ \\
\cline { 2 - 3 } Transmissão & 5. A AIDS é uma doença que tem cura? & $66 \%$ \\
\hline & 6. O vírus da doença AIDS pode ser transmitido por abraço ou beijo no rosto? & $92 \%$ \\
\cline { 2 - 3 } & 7. O vírus da AIDS pode ser transmitido por picada de mosquito? & $54 \%$ \\
\cline { 2 - 3 } & $\begin{array}{l}\text { 8. O vírus da AIDS pode ser transmitido pelo uso de seringas e agulhas } \\
\text { compartilhadas? }\end{array}$ & $99 \%$ \\
\hline \multirow{2}{*}{ Prevenção } & $\begin{array}{l}\text { 9. O uso de camisinha nas relações sexuais impede a transmissão do vírus da } \\
\text { AIDS? }\end{array}$ & $90 \%$ \\
\hline
\end{tabular}

Tabela 2. Porcentagem de acerto sobre HIV relacionado com as três dimensões de perguntas

\begin{tabular}{c|c|c|c}
\hline $\begin{array}{c}\text { Nível de conhecimento } \\
\text { geral }\end{array}$ & $\begin{array}{c}\text { Conhecimento } \\
\text { geral }\end{array}$ & Transmissão & Prevenção \\
\hline Insuficiente & $40 \%$ & $67 \%$ & $68 \%$ \\
\hline Suficiente & $81 \%$ & $85 \%$ & $94 \%$ \\
\hline
\end{tabular}

Sobre a prevenção pessoal, 29 entrevistados responderam que fazem uso de camisinha nas relações sexuais (25\%), e 86 entrevistados responderam que não fazem uso da mesma $(75 \%)$.
Embora a média de acertos de quem usa camisinha é maior do que os respondente que não usa, não existem diferenças estatísticas pelo teste $\mathrm{F}$ entre a média dos fazem uso ou não $(\mathrm{p}=0,129)$ (Gráfico 1). 
Gráfico 1. Número de acertos nas categorias "conhecimento geral" e "transmissão" vs uso de preservativo

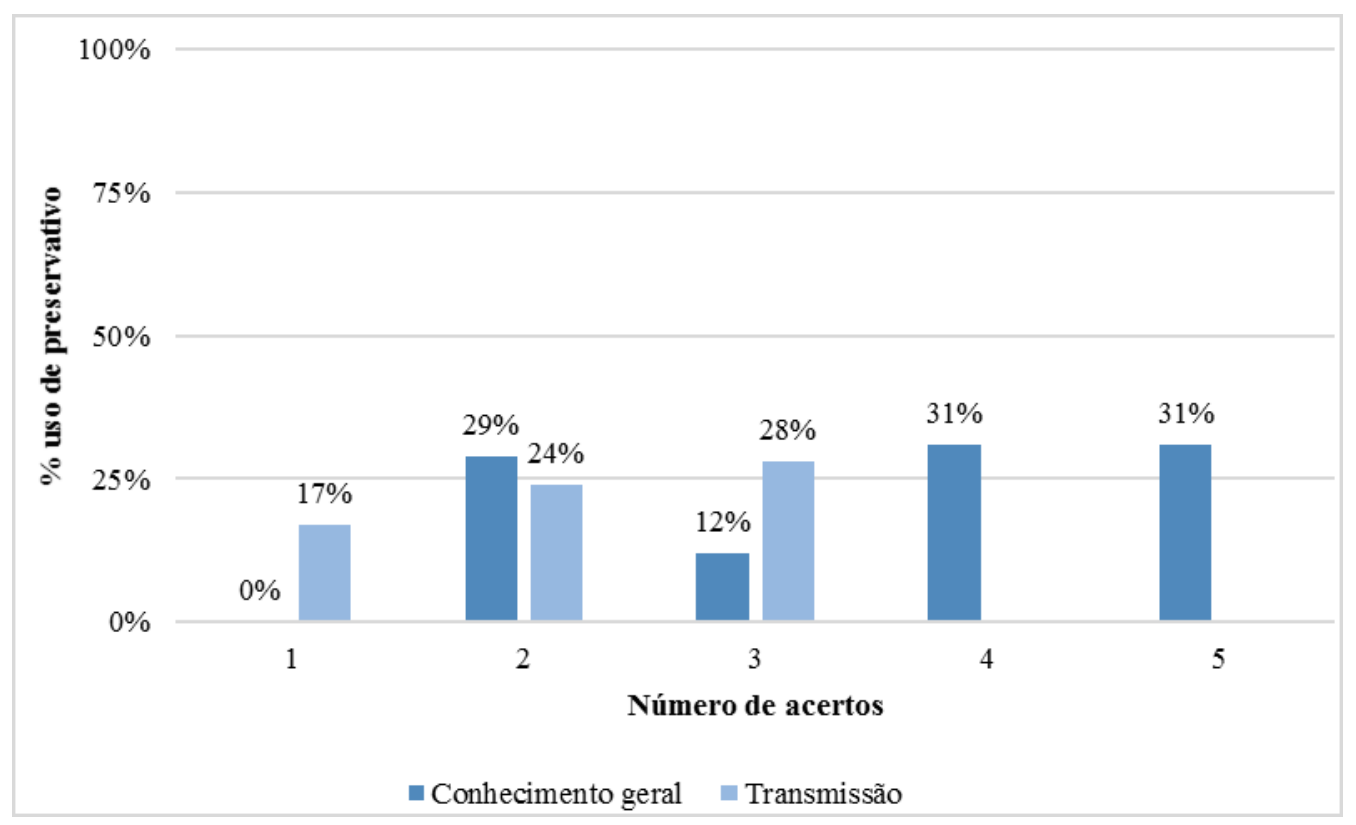

Notou-se também que quanto maior a taxa de acertos nas questões sobre transmissão, maior o uso do preservativo pelo respondente (Gráfico 1).

Avaliando o uso de preservativos com a quantidade de acertos nas subcategorias "conhecimento geral" e "transmissão", observa-se uma tendência de aumento do uso de preservativos em relação a um maior número de acerto de questões, porém este dado não apresentou significância estatística $(\mathrm{p}=0,4553)$.

\section{DISCUSSÃO}

Os respondentes da pesquisa foram em sua maioria mulheres $(68,7 \%)$, fato que reforça a realidade de que a busca por serviços médicos é mais prevalente no sexo feminino. Segundo Lemos ${ }^{7}$, os homens ainda relacionam a doença à fragilidade e vulnerabilidade, não como condição biológica que, junto aos valores culturais, sociais e até mesmo de desinformação fazem com que a população masculina não procure atendimento de saúde preventivamente.

Das três esferas presentes nesse estudo: conhecimento, transmissão e prevenção, o domínio do conhecimento sobre o HIV foi a mais deficitária. A maior defasagem foi sobre a presença de sintomas nos indivíduos com HIV, representando apenas 44\% de acerto. Em Melo ${ }^{8}$, as respostas insatisfatórias sobre os conhecimentos gerais do HIV/AIDS predominaram entre os idosos, assim como no presente estudo. Apesar de ser mais defasado o conhecimento sobre a doença, comparativamente às outras áreas, em ambos os sexos a taxa de acerto nas questões sobre conhecimento da doença foi de $74 \%$, fato que é discutido também por Bastos et al. ${ }^{2}$, em que dois terços dos entrevistados tinham um entendimento mínimo sobre a doença. Em um aspecto geral, apesar de ambos os estudos avaliarem positivamente o conhecimento do idoso (acima de $60 \%$ ), esse fator ainda não é suficiente para diminuir a incidência da doença nessa faixa etária.

Foi apresentado um alto conhecimento no quesito transmissão, contudo, a grande porcentagem de erro na pergunta sobre a transmissão do HIV por picada de mosquito demonstra que ainda há defasagem no conhecimento sobre transmissão da doença. De maneira semelhante, em Monteiro ${ }^{9}$, uma parte considerável respondeu que a transmissão por picada de mosquito era possível $(25 \%)$.

Evidenciou-se que independente do nível de conhecimento, os indivíduos possuem entendimento sobre a prevenção de HIV. Isso pode estar relacionado com a facilidade de acesso a informação disponível atualmente. Diferentemente, em Brito et al. ${ }^{10}$, foi avaliado o conhecimento sobre o HIV/AIDS de idosos orientados e uma parcela dos participantes não apontou a utilização do preservativo como forma de prevenção.

Quanto à prevenção pessoal, 25\% dos entrevistados responderam que fazem o uso de camisinha nas relações sexuais, enquanto que $75 \%$ não fazem o uso dessa. Tal fato pode ser justificado pela maioria dos entrevistados serem mulheres sem vida sexual ativa ou possuírem um(a) parceiro(a) fixo(a) e acreditarem não ser necessária a prevenção, dado que grande parte das entrevistadas utilizavam essa justificativa ao responder que não faziam uso do preservativo nas relações sexuais, apesar desses dados não terem sido quantificados.

Constatou-se, com o presente estudo, que embora o "conhecimento geral" sobre HIV/AIDS tenha tido 
uma porcentagem significativa de acertos ainda existe desinformação quanto a alguns aspectos da doença, tais como: a crença do HIV ser transmitido por picada de mosquito (54\%) e a existência de cura para AIDS (66\%).

Além disso, identificou-se menor taxa de acerto no conhecimento geral de HIV/AIDS comparativamente às outras áreas analisadas e levando em consideração que essas informações são transmitidas em sua grande maioria por meios de comunicação social como televisão, rádio e jornais ${ }^{11}$ torna-se considerável que o conhecimento sobre ISTs não seja difundido majoritariamente por esses meios. É necessário, portanto, que a saúde pública e profissionais de saúde busquem ampliar a compreensão do processo de expansão da AIDS nessa faixa etária para execução de estratégias de educação do idoso, fazendo com que a população acima de 60 anos entenda como a doença funciona, seus mecanismos de transmissão e prevenção e da mesma forma, que o profissional se conscientize do atual quadro de aumento de ISTs na terceira idade.

Sugere-se a necessidade de ampliar as discussões sobre a temática, abordando a diversidade social, econômica e cultural dos respondentes em estudos futuros, com amostras maiores e igualmente representadas pelos sexos feminino e masculino, realizando concomitantemente a educação do idoso sobre HIV/AIDS após a coleta de dados da entrevista.

É importante salientar que as limitações desta pesquisa referem-se ao número amostral, sugerindo que o grupo não é representativo da cidade de São Paulo; respondentes predominantemente do sexo feminino.

Participação dos autores: Liberali BM: planejamento do projeto de pesquisa, aplicação do questionário, coleta e análise de dados, redação do estudo, referências. Neves SCM: planejamento do projeto de pesquisa, aplicação do questionário, coleta de dados e redação do estudo. Oliveira LS: planejamento do projeto de pesquisa, aplicação do questionário e redação do estudo. Batista BD: planejamento do projeto de pesquisa, aplicação do questionário, redação do estudo, título em inglês e abstract. Nacaratto DCFF: revisão do texto e orientações gerais. Cavazzana CL: orientações iniciais, planejamento e revisão do projeto de pesquisa.

\section{REFERÊNCIAS}

1. Campos ACV, Gonçalves LHT. Aging demographic profile in municipalities in the state of Pará, Brazil. Rev Bras Enferm. 2018;71(Supl. 1):636-43. doi: http://dx.doi.org/10.1590/00347167-2017-0070.

2. Bastos LM, Tolentino JMS, Frota MAO, Tomaz WC, Fialho MLS, Batista ACB, Teixeira AKM, Barbosa FCB. Avaliação do nível de conhecimento em relação à Aids e sífilis por idosos do interior cearense, Brasil. Ciên Saúde Coletiva. 2018;23(8):2495-502. doi: http://dx.doi.org/10.1590/141381232018238.10072016 .

3. Brasil. Ministério da Saúde. Secretaria de Vigilância em Saúde. HIV/Aids 2018. Bol Epidemiol. 2018;49(53):166. [citado 5 ago. 2019]. Disponível em: file://C:/Users/ Administrador/Downloads/boletim_hiv_aids_12_2018.pdf.

4. Brasil. Ministério da Saúde. Secretaria de Vigilância em Saúde. Aids e DST 2006. Bol Epidemiol AIDS/DST. 2006;III(1). [citado 7 ago. 2019]. Disponível em: file:///C:/ Users/Administrador/Downloads/boletim_epidemiologico_ aids_e_dst_-_2006.pdf.

5. Souza MHT, Backes DS, Pereira AD, Ferreira CLL, Medeiros HMF, Marchiori RCT. Nivel de conocimiento sobre el Síndrome de Inmunodeficiencia Adquirida en un grupo de adultos mayores. Av Enferm (Bogotá). 2009;27(1):22-9. https://revistas.unal.edu.co/index.php/avenferm/article/ view/12950.

6. Sousa ACA, Suassuna DS, Costa SM. Perfil clínicoepidemiológico de idosos com Aids. DST J Bras Doenças
Sex Transm. 2009;21(1):22-6. Disponível em: http:// www.dst.uff.br/revista21-1-2009/5-Perfil\%20ClinicoEpidemiologico-\%20JBDST\%2021(1)\%202009.pdf.

7. Lemos AP, Ribeiro C, Fernandes J, Bernardes K, Fernandes R. Saúde do homem: os motivos da procura dos homens pelos serviços de saúde. Rev Enferm UFPE. 2017;11(Supl. 11):454653. doi: 10.5205/reuol.11138-99362-1-SM.1111sup201714.

8. Melo HMA, Leal MCC, Marques AP de O, Marino JG. O conhecimento sobre aids de homens idosos e adultos jovens: Um estudo sobre a percepção desta doença. Cienc Saude Coletiva. 2012;17(1):43-53. doi: http://dx.doi.org/10.1590/ S1413-81232012000100007.

9. Monteiro TJ, Trajano LASN, Carvalho DS, Pinto LAP, Trajano ETL. Avaliação do conhecimento sobre HIV/AIDS em grupo de idosos através do QHIV3I. Geriatr Gerontol Aging. 2016;10(1):29-33. doi: http://dx.doi.org/10.5327/ Z2447-2115201600010006.

10. Brito NMI, Andrade SSC, Silva FMC, Fernandes MRCC, Brito KKG, Oliveira SHS. Idosos, Infecções Sexualmente Transmissíveis e aids: conhecimentos e percepção de risco. ABCS Heal Sci. 2016;41(3):140-5. doi: http://dx.doi. org/10.7322/abcshs.v41i3.902.

11. Laroque MF, Affeldt AB, Cardoso DH, Souza GL, Santana MG, Lange C. Sexualidade do idoso: comportamento para a prevenção de DST/AIDS. Rev Gaucha Enferm (Porto Alegre). 2011;32(4):774-80. doi: http://dx.doi.org/10.1590/ S1983-14472011000400019.

Recebido: 03.12.2019

Aceito: 30.01 .2020 